\title{
Histology and histochemistry of sea anemones in environmental contamination studies
}

\author{
J. R. Gadelha***, F. Morgado* and A.M.V.M. Soares* \\ *CESAM \& Departamento de Biologia, **CAPES Foundation, Ministry of Education of Brazil, Brasília - DF \\ 70040-020, Brazil.
}

Since contaminants such as metals, POPs (Persistent Organic Pollutants) and PAHs (Polycyclic aromatic compounds represent a risk to human health and to the environment, it is therefore extremely necessary to study their biological effects $[1,2,3]$. Man-made chemicals endocrine disruptors such as estrogens pose the potential to modulate endocrine function and thus adversely affect humans and other animal's reproductive development. In this work, sublethal toxicity tests were carried out with the sea anemones Actinia equina and Anemonia sulcata exposed to 17- $\beta$-Estradiol. A. equina and $A$. sulcata are species that present a wide geographic distribution and might possibly be effective pollution indicators. Histological and histochemical techniques were used to detect morphological changes in sea anemones in order to find histological parameters that could be useful as early biomarkers of environmental contamination [3]. The histological and histochemical procedures followed by standard methods to Hematoxylin and eosine (H \& E), Periodic acid Shiff reagent (PAS) and Masson`s Tricrome (TMass) stained adaptated to Actiniidae conditions. Such as, the fixation (formalin and alcohol) time is high (96 hours), because this organisms are almost $98 \%$ of water body constitution. The slides obtained were observed by light microscopy means. The assemblage of methodologies described permitted the identification of several anomalies/pathologies in different parts of the sea anemones body, with special attention to reproductive structures. Results obtained for $A$. sulcata showed vitellogenic oocytes with anomalous dimensions, altered cytoplasm or without cellular membrane limits (Figure 1). It can also be observed lipid accumulations and cells membranes not always preserved. In certain areas oocytes presents small reactivity with atypical PAS low basophilic patterns. In the mesoglea the amoebocytes showed more eosinophilic cytoplasm or extracellular bodies suggesting necrosis or protein content. The effects at $10 \mu \mathrm{g} / \mathrm{L}$ concentrations show a considerable number of oocytes with germinal vesicles membranes and indistinct cytoplasm boundaries (Figure 1). Results obtained for A. equina showed some morphological changes in the spermatocytes of male gonads and in the germinal vesicles the female gonads. The effects observed at higher concentrations shows oocytes and ovarian tissues disintegration. The morphologic alterations observed suggested a delay in spermatogenesis [4] and although there have been no alterations in female vitellogenic granules, there are changes in their maturation. The whole effects lead to verify the role that the estradiol in the Anthozoan reproductive system.

\section{References}

1. Chang L.W. et al.,Toxicology of Metals. Boca Raton: Lewis Publishers, 1996.

2. Atkinson S. and Atkinson M.J., Coral Reefs, 11:33-35, 1992.

3. Pinder L.C.V. et al., Endocrine Function in Aquatic Invertebrates and Evidence for Disruption by Environmental Pollutants. 8 Environment Agency. ISBN: 1-873160-78-X. 1999.

4. Tay K.L. et al., Environmental Health Perspectives, 111:273, 2003.

Acknowledgments: This work was supported by the Portuguese Foundation for the Science and Technology - Portugal and FEDER funds, through the Projects: PTDC/MAR/464729/2006 and FCT/CNPq (Brazil), Project 6818, Programme 19/ 004. 


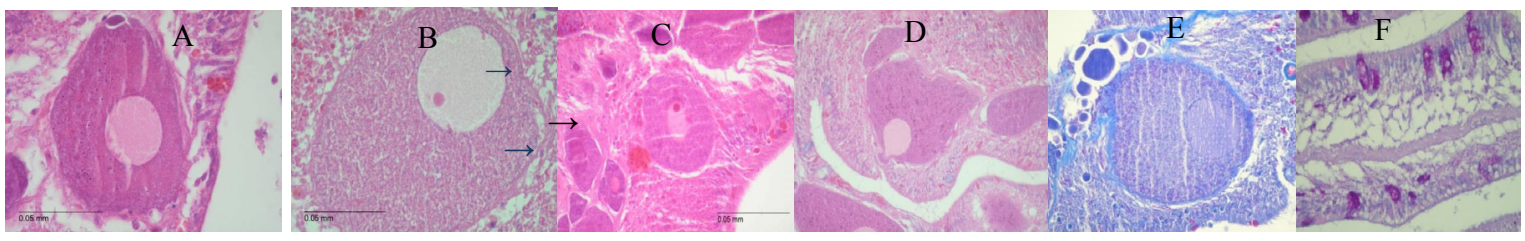

Figure 1. Sublethal 17-B-estradiol exposed to 96 hours on Actinia equina under control, 10, 100, $200 \mu \mathrm{g} / \mathrm{L}$. The appearance of some gonadal (H \& $\mathrm{E}$ preparations) A- Control seawater; B- Control solvent (vitellogenic oocytes), C- Concentration $10 \mu \mathrm{g} / \mathrm{L}: \quad$ A. sulcata ovaries, nuclear oocytes membrane with indistinct boundaries $\quad(\rightarrow)$, and primary oocytes $(\rightarrow)$; $\quad$ D-Concentration $\quad 100 \mu \mathrm{g} / \mathrm{L}$ (vitellogenic oocyte with irregular morphology), E-Vittelogenic oocytes with peripheral nuclei and visible nucleoli and oocyte in the first stage of vitellogenesis with cell membrane and irregular indistinct boundaries; FConcentration $200 \mu \mathrm{g} / \mathrm{L}$ - Mesentery detail, with no effects at mucosal cells level and possible appearance of lipids accumulation areas (Bar: $0,05 \mathrm{~mm})$

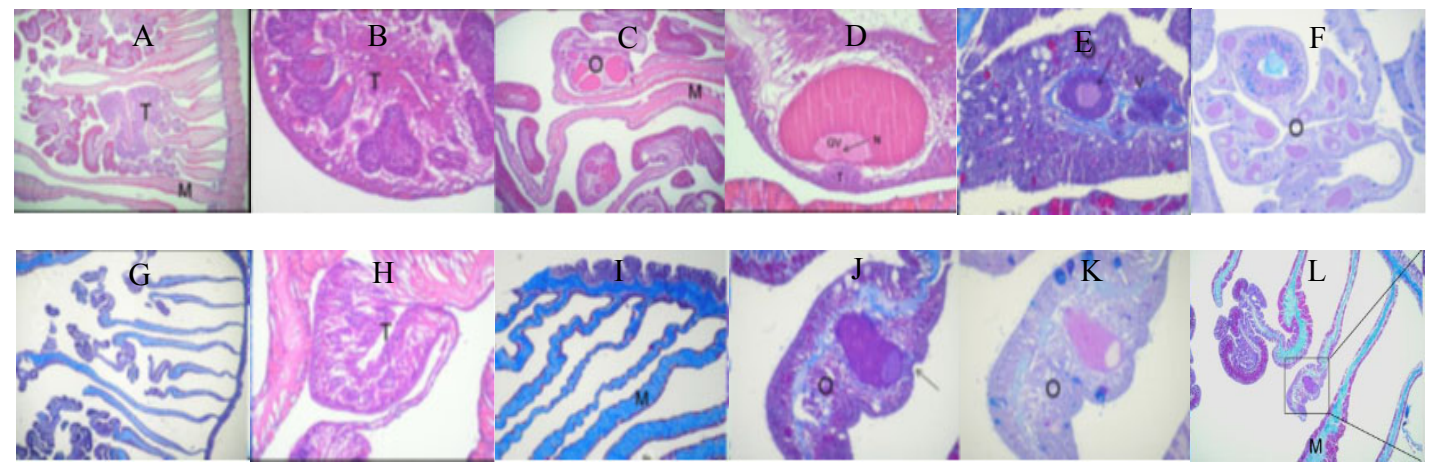

Figure 2. A, B, C and D with hematoxylin and eosine colorations controls: male gonads and mesenteries; BNormal male gonad; C- Fertile female mesentery; D- Vitellogenic oocyte; E and F concentration 10 $\mu \mathrm{g} / \mathrm{L}$ : EOvary with vitellogenic oocyte showing irregularities in the nuclear membrane (Mass $\mathrm{T}$ ) and FOvaries with mature oocyte (APAS); G and H concentration $100 \mu \mathrm{g} / \mathrm{L}$ : G- mesenteries, with ovaries in early stages of development (TMass) and H-Testis with anatomical changes (H \& E) (L:Lise and T: Testis); I, J, K and L Concentration $200 \mu \mathrm{g} / \mathrm{L}$ : I- mesenteries infertile (TMass), J- oocyte with visible trophonema (TMass), KOvary with mucous cells as evidenced by the A / PAS and L- Ovaries (TMass) (Bar: 0,01mm). 